\section{ORIGINAL RESEARCH}

S.M. Lim

I.S. Choi

B.A. Hum

C.A. David

\title{
Dissecting Aneurysms of the Distal Segment of the Posterior Inferior Cerebellar Arteries: Clinical Presentation and Management
}

BACKGROUND AND PURPOSE: Dissecting aneurysms of the distal segment of the PICA are rare. The purpose of this study was to evaluate the clinical presentations, imaging features, treatment options, and clinical outcomes of dissecting PICA aneurysms.

MATERIALS AND METHODS: Six patients with dissecting aneurysms in the distal segments of PICA were found in the database of a single medical center, from November 1996 to December 2008, and retrospectively evaluated. Treatment mode and follow-up clinical outcomes were analyzed.

RESULTS: Five patients with dissecting PICA aneurysms presented with acute intracranial hemorrhage and 1 patient presented with a large mass from an intramural hematoma. All 5 patients with intracranial hemorrhage were treated with endovascular occlusion of both the dissecting PICA aneurysm and the distal parent artery. The patient with the intramural hematoma underwent surgical trapping with end-to-end anastomosis. In 1 patient, the dissecting aneurysm recurred twice within a 5 year 3 month period, despite endovascular occlusion of both the aneurysm and the parent artery. The clinical outcome postprocedure was excellent in all patients, without permanent neurologic complication.

CONCLUSIONS: For the endovascular treatment of dissecting aneurysms in the distal PICA segments, we recommend occlusion of both the dissecting aneurysm and the parent artery to avoid leaving the point of initial intimal tear untreated. All of our patients had excellent clinical outcomes; however, our experience with recanalization illustrates the need for close follow-up of patients.

ABBREVIATIONS: PICA = posterior inferior cerebellar artery; $\mathrm{SAH}=$ subarachnoid hemorrhage

D issections and dissecting aneurysms of the cerebrovascular system are now more frequently diagnosed, due to a better understanding of the underlying pathophysiology, as well as to advances in neuroimaging, specifically MR imaging and MR angiography. ${ }^{1,2}$ Most spontaneous dissections involve the extracranial carotid and vertebral arteries, often causing pain in the neck or skull base at onset, but otherwise they demonstrate few clinically significant symptoms. Many of these dissections likely go unrecognized, and thus the true incidence is not well documented. The dissections recognized in clinical practice usually present with more serious symptoms, such as severe neck pain or a thromboembolic event. Only a small percentage of dissections of the brachiocephalic arteries involve the intracranial arteries, resulting in spiral segmental stenosis or intracranial hemorrhage. Intracranial dissections causing SAH occur more often in the vertebrobasilar system compared with the carotid arteries. ${ }^{3}$ An isolated dissection of the distal segment of the PICA with aneurysm formation is a very rare occurrence, accounting for only $0.5 \%-0.7 \%$ of all intracranial aneurysms. ${ }^{4}$

Tawk et $\mathrm{al}^{5}$ reviewed all published cases of isolated dissecting PICA aneurysms and reported only 27 cases from 1966 to

Received September 11, 2009; accepted after revision November 20.

From the Department of Radiology (S.M.L.), Ewha Womans University Hospital, Seoul, Korea; and Departments of Radiology (I.S.C., B.A.H.) and Neurosurgery (C.A.D.), Lahey Clinic Medical Center, Burlington, Massachusetts.

Please address correspondence to In Sup Choi, MD, Lahey Clinic Medical Center, 41 Mall Rd, Burlington, MA 01805; e-mail: in.sup.choi@lahey.org

Indicates open access to non-subscribers at www.ajnr.org

DOI 10.3174/ajnr.A2014
2001. SAH was the most common initial clinical presentation (74\%), with ischemia accounting for the remaining cases. The anatomy of the PICA and the location of the dissection are variable among the individual patients.

All of the publications that we know of on dissecting PICA aneurysms include only a small number of cases. Treatment decisions were based on the individual clinician's experience, without a standardized approach to treatment. Most reported cases have been treated by surgery, with direct clipping, wrapping, or trapping with or without bypass. ${ }^{6}$ More recently, several small series or case reports have been published that illustrate successful endovascular management. Endovascular treatment decreases the risks associated with manipulating the brain stem and cranial nerves during surgery. ${ }^{7-9}$ The endovascular approach in most cases occluded the parent artery near or at the level of the dissecting aneurysm. However, Cellerini et $\mathrm{al}^{11}$ and others reported coil treatment of the dissecting aneurysm sacs without parent artery occlusion, claiming it would prevent any chance of PICA territory infarct. ${ }^{5,10,11}$

\section{Materials and Methods}

From the database of all patients who underwent diagnostic angiography or endovascular treatment for intracranial vascular lesions at a single center, from November 1996 until December 2008, 25 angiographically confirmed PICA aneurysms were identified. Six were dissecting aneurysms of the distal PICA segment. All typical saccular aneurysms at the PICA origin and PICA aneurysms with associated findings of irregular fusiform shape and narrowing of the vertebral artery, suggesting that the aneurysm was likely from extension of a vertebral artery dissection, were excluded.

The mean age of the patients ( 4 women, 2 men) was 57.9 years, ranging from 28 to 80 years old. Pretreatment clinical status in those 


\begin{tabular}{|c|c|c|c|c|c|c|c|}
\hline \multicolumn{8}{|c|}{ Characteristics of 6 patients with peripheral dissecting aneurysms of the PICA } \\
\hline $\begin{array}{l}\text { Case } \\
\text { No. }\end{array}$ & Age/Sex & $\begin{array}{l}\text { Hunt and } \\
\text { Hess Grade }\end{array}$ & Initial CT and MR Imaging Findings & $\begin{array}{l}\text { Location of } \\
\text { Aneurysm }\end{array}$ & Treatment & $\begin{array}{l}\text { Recurrent } \\
\text { Aneurysm }\end{array}$ & Outcome \\
\hline 1 & $50 / F$ & & Intracerebellar hematoma & Right telovelomedullar & Endovascular & Yes & Excellent \\
\hline 2 & $62 / F$ & & Mural hematoma & Left lateral medullary & Surgery & No & Excellent $^{\mathrm{a}}$ \\
\hline 3 & $65 / M$ & 2 & Subarachnoid hemorrhage, intraventricular hemorrhage & Left tonsilomedullary & Endovascular & No & Good \\
\hline 4 & 28/M & 5 & $\begin{array}{l}\text { Subarachnoid hemorrhage, subdural hematoma, } \\
\text { contusion }\end{array}$ & Left anterior medullary & Endovascular & No & Good \\
\hline 5 & $35 / F$ & 3 & Subarachnoid hemorrhage, intraventricular hemorrhage & Right lateral medullary & Endovascular & No & Excellent \\
\hline 6 & $80 / F$ & 1 & Subarachnoid hemorrhage, intraventricular hemorrhage & Left anterior medullary & Endovascular & No & Excellent \\
\hline
\end{tabular}

${ }^{a}$ Died of renal cell carcinoma 1 year after treatment of dissection.
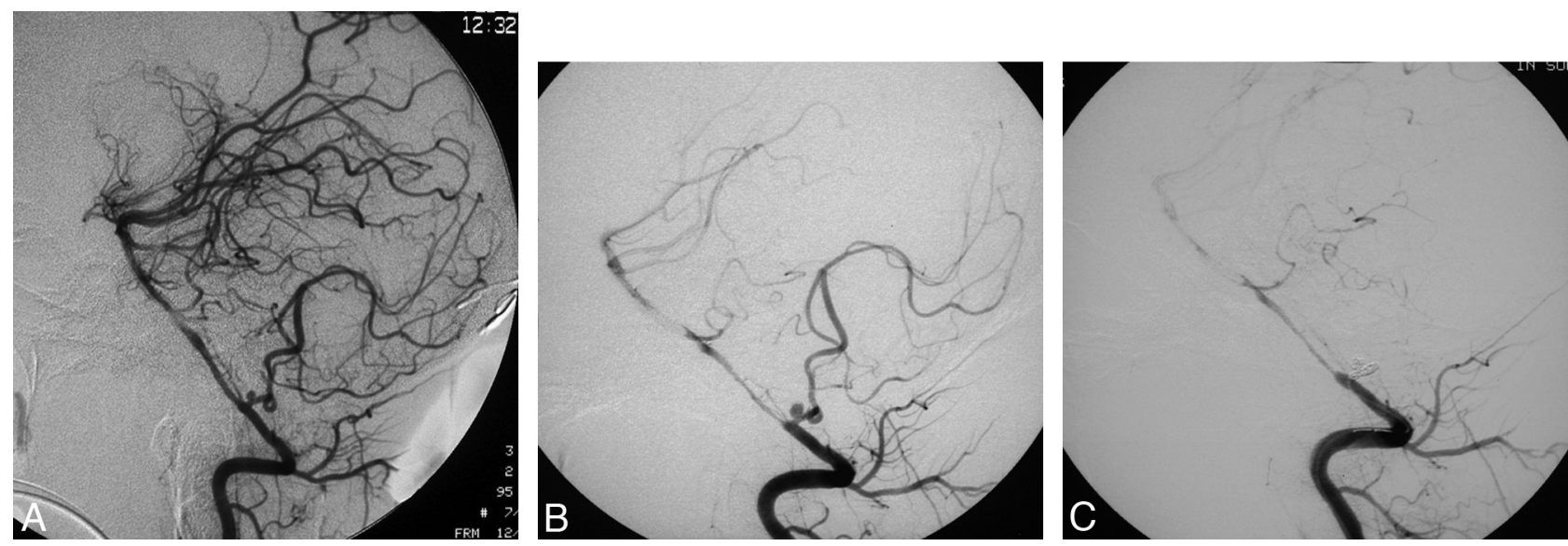

Fig 1. A 28-year-old man (patient 4) had serious head trauma from motor vehicle crash. $A$, The cerebral angiogram following the second SAH shows a small aneurysm at the anterior medullary segment of left PICA. B, Selective angiogram of the left vertebral artery at the time of treatment illustrates the PICA aneurysm increased in size in a day and diffuse spasm of the vertebral and basilar artery. C. Postembolization injection of the left vertebral artery demonstrates complete occlusion of the PICA at its origin with coils in the patent artery and the aneurysm.

patients who presented with SAH was recorded according to the Hunt and Hess classification. ${ }^{12}$ The time to treatment ranged from 1 day to 10 days (mean, 3.5 days). All angiography and endovascular procedures were performed in a neuroangiography suite equipped with digital subtraction angiography (BN 3000; Philips Medical Systems, Best, the Netherlands) under general anesthesia. Three-dimensional rotational angiography was performed in 4 patients. In all patients, a complete diagnostic angiogram was obtained before endovascular treatment to define the details of the vascular anatomy of the posterior fossa and assess underlying vascular disease.

Dissection was diagnosed if the lesion demonstrated irregular fusiform dilation with or without associated narrowing of the PICA. ${ }^{10}$ The location of the aneurysm was recorded according to the classification of segments described by Lister et al, ${ }^{13}$ including: 1) anterior medullary, 2) lateral medullary, 3) tonsillomedullary, 4) telovelotonsillar, and 5) cortical segments. Among the patients selected for endovascular treatment, the aneurysms and proximal parent arteries were coiled by using Guglielmi detachable coils (Boston Scientific, Fremont, California) or Orbit Trufill coils (Cordis, Miami Lakes, Florida). In cases of a small dissecting aneurysm, the tip of the microcatheter was positioned proximal to the dissecting aneurysm, and the initial loop of the first coil was made in the parent artery and then pushed into the pseudoaneurysm sac, eventually embolizing both the dissecting aneurysm and proximal segment of the parent artery. Procedure-related complications and adverse events were recorded.

After the procedure, the patients were closely monitored in an intensive care unit for at least 1 day. Postprocedure CT scans were obtained in all patients within 24 hours. A follow-up CT scan (1 pa- tient), follow-up MR imaging/MR angiography studies (4 patients), and follow-up angiograms (3 patients) were analyzed for recurrent aneurysm or new intracranial abnormality.

The final outcome was assessed at the last clinical visit or phone interview. All data concerning the demographics, clinical presentation, imaging findings, procedures, and clinical outcomes are presented in the Table.

\section{Results}

Five patients presented with an acute intracranial hemorrhage (4 patients with SAH and intraventricular hemorrhage and 1 patient with intracerebellar hemorrhage). Before treatment, the Hunt and Hess grades of the patients with SAH were 1, 2, 3, and 5.

Patient 4 (Fig 1) had serious head trauma from a motor vehicle crash. The initial CT scan showed a small amount of blood in the lateral ventricle, fourth ventricle, and foramen magnum, a subdural hematoma in the falx cerebri, and a hemorrhagic contusion in the right frontal lobe. The SAH was thought to be traumatic in origin. Seven days after admission, the patient's neurologic status deteriorated. A follow-up CT scan showed recurrent SAH. The cerebral angiogram demonstrated a small aneurysm in the anterior medullary segment of the left PICA. Additionally, it showed a traumatic pseudoaneurysm of the left superficial temporal artery. The patient was transferred to our institution the next day for endovascular treatment. A repeat angiogram showed that the PICA aneurysm had tripled in size. The aneurysm and parent artery were successfully occluded with detachable coils. 

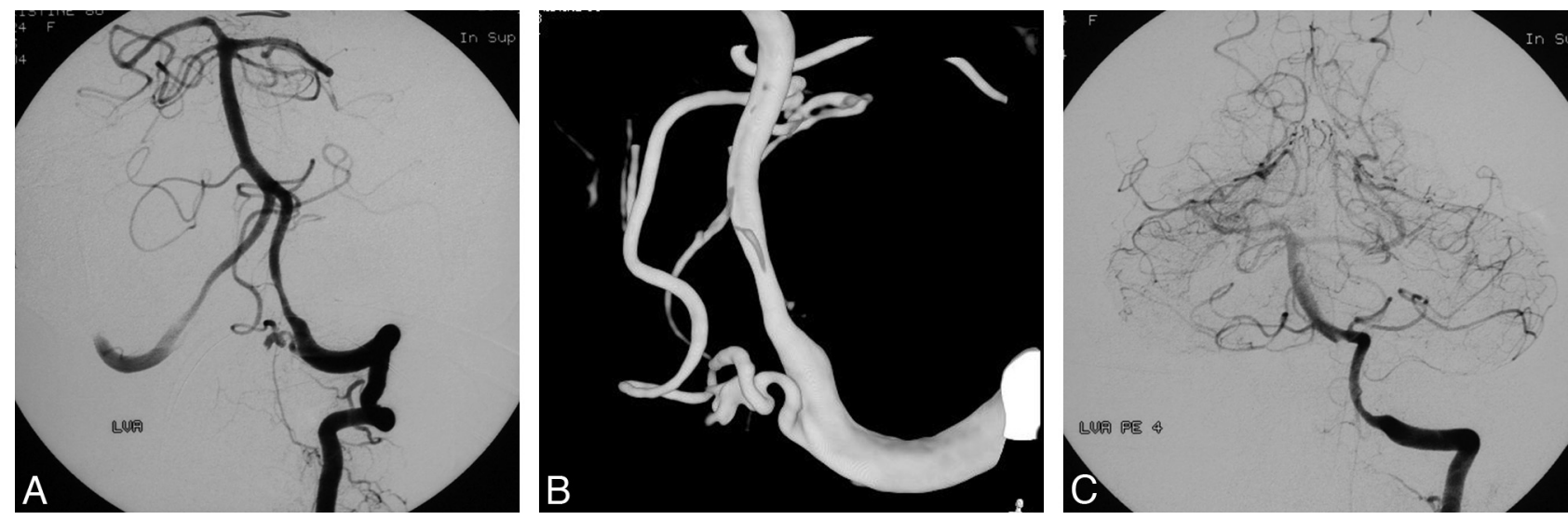

Fig 2. An 80-year-old woman (patient 6) presented with sudden severe headache. $A$ and $B$, Initial digital subtraction angiogram of the left vertebral artery in the frontal projection and $3 \mathrm{D}$ rotational angiogram show a fusiform irregular-shaped aneurysm at the anterior medullary segment with focal irregularity of left PICA, indicating dissection. $C$, The postembolization angiogram illustrates the dissecting aneurysm and parent artery are occluded.

Patient 2 complained of constant occipital headache. MR imaging showed a large, well-circumscribed hematoma anterior to the cerebellar tonsils, without SAH. The subsequent cerebral angiogram revealed a small fusiform aneurysm from the lateral medullary segment of the left PICA with focal narrowing of the parent artery proximal to the aneurysm, highly suggestive of dissection, with a large intramural hematoma. The left PICA was dominant and demonstrated poor washout in the superselective injection, indicating insufficient collateral circulation. Thus, it was decided to treat the patient surgically with trapping and end-to-end anastomosis of the left PICA, which was successfully performed without complication.

The remaining 4 patients presented with spontaneous rupture of their aneurysms; 3 presented with SAH and 1 with an intracerebellar hematoma. Selective angiograms demonstrated fusiform widening with narrowing of the PICA trunk proximal to the aneurysm lumen, a typical appearance for dissection.

Four of the aneurysms were on the left side (67\%). The locations of the aneurysms along the PICA included the anterior medullary segment in 2 cases $(33.3 \%)$, the lateral medullary segment in 2 cases $(33.3 \%)$, the tonsilomedullary segment in 1 case $(16.7 \%)$, and the telovelomedullary segment in 1 case (16.7\%). In 3 patients, the PICAs were symmetric in size; 1 patient had a relatively small caliber PICA at the lesion site, and 2 patients had dominant PICAs at the dissecting site.

The maximal diameter of the aneurysms ranged from 2 to $12 \mathrm{~mm}$ (mean, $4.5 \mathrm{~mm}$ ). Rotational angiograms (Fig 2) showed the irregular fusiform shapes of the dissecting aneurysms and focal irregularity of the PICAs.

Two patients with symmetric PICAs had small ipsilateral PICA territory infarcts on follow-up MR imaging. However, these patients did not show clinical signs of ischemia following occlusion of the PICA trunks. No other incidental aneurysms or underlying vascular lesions were detected in the remaining intracranial arteries.

The endovascular treatment was performed with occlusion of the dissecting aneurysm and distal segment of the PICA proximal to the aneurysm in all patients. At the end of each procedure, occlusion of the aneurysm and distal parent artery was confirmed by a selective angiogram. No immediate pro- cedure-related complications, such as aneurysm rupture or coil migration, occurred. Posttreatment CT scans within 24 hours demonstrated no additional abnormality.

Follow-up angiograms showed 2 cases (33.3\%) of recanalization of the occluded parent arteries. In patient 1 , a final angiogram confirmed that the PICA trunk was completely occluded at the tonsillomedullary segment. Follow-up angiograms at 6 months and at 1 year 10 months showed recanalization of the PICA without visualization of the dissecting aneurysm. However, 4 years after initial endovascular treatment, the patient had an episode of severe headache. MR imaging showed recurrent intracerebellar hemorrhage around the coil mesh, but no SAH. This patient was immediately retreated for recurrent dissection of the recanalized PICA. Three months after the second treatment, the patient experienced a similar pattern of occipital headache. Dissection was again seen at the trunk of the PICA, proximal to the point of occlusion from the second treatment, with a large aneurysmal lumen. The aneurysm was endovascularly retreated a third time without subsequent recurrence for 2 years.

In patient 5, a follow-up angiogram 20 months after initial treatment (Fig 3) showed recanalization of the occluded PICA; however, the dissecting aneurysm remained occluded, without recurrence on the 5-year follow-up angiogram. In the remaining patients, there was no change on follow-up angiograms or MR imaging.

Follow-up imaging ranged from 3 months to 5 years 9 months in 5 patients. One patient (patient 2) died from renal cell carcinoma 1 year after the surgery of the dissecting aneurysm. The postprocedure clinical outcomes were otherwise good to excellent in all patients.

\section{Discussion}

PICA dissecting aneurysms account for $\sim 0.5 \%$ to $0.7 \%$ of all intracranial aneurysms, ${ }^{4}$ with most located at the origin or in the first anterior medullary segment of the vessel. ${ }^{14}$ After excluding aneurysms at the PICA origin, the location of our cases included $67 \%$ in the anterior or lateral medullary segments and $67 \%$ on the left side. Among distal dissecting PICA aneurysms in the literature, the dissecting etiology has been found in $0 \%-80 \%$ of the cases. ${ }^{15-17}$ In our cases, the etiology of the 

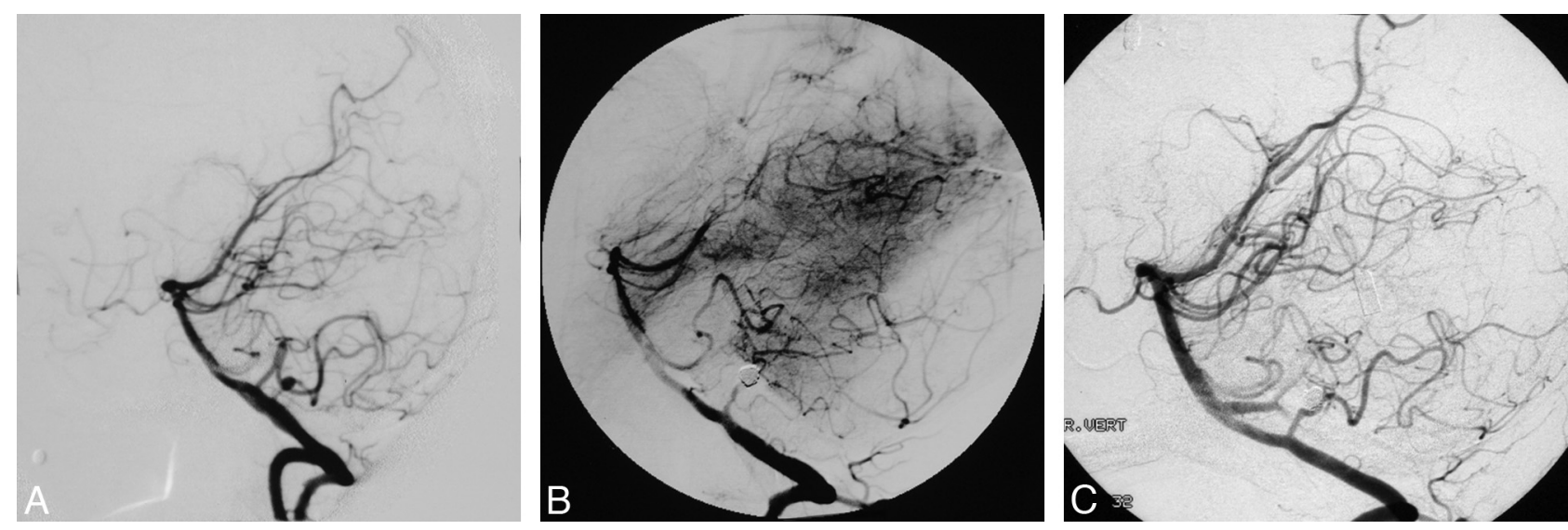

Fig 3. A 35-year-old woman (patient 5) presented with Hunt and Hess grade III SAH. A, A lateral projection of the right vertebral angiogram demonstrates a $3 \times 4 \mathrm{~mm}$ fusiform aneurysm at the lateral medullary segment of right PICA. $B$, The aneurysm is treated by endovascular occlusion of dissecting aneurysm and parent artery. The postocclusion angiogram in the lateral projection shows complete obliteration of the PICA trunk and the aneurysm. $C$, Five-year follow-up DS angiogram; note that the occluded PICA is recanalized without any growth of the aneurysm.

dissection was presumed to be spontaneous in 5 cases and traumatic in 1 case.

The natural history of distal segment dissecting PICA aneurysms is likely different from the more common saccular aneurysms, which frequently arise at the vertebral arteryPICA junction. ${ }^{11}$ One author reported a high rebleeding risk $(24 \%)$ in the acute phase of the posterior circulation dissections, with an associated high mortality rate. ${ }^{18}$ We had 1 patient $(17 \%)$ with rebleeding in the acute phase who was treated when the second hemorrhage occurred. The patient recovered without neurologic deficit except from a frontal lobe contusion from concurrent trauma.

Dissecting aneurysms were diagnosed angiographically if the lesion initially demonstrated an irregular fusiform dilation with or without associated narrowing of the PICA. We excluded the irregular saccular-shaped aneurysms located at the PICA origin, as those aneurysms may be an extension of a vertebral artery dissection.

Management remains controversial. Isolated case reports have described the treatment of these lesions with wrapping or with the sacrifice of the PICA with or without distal revascularization. ${ }^{19-21}$ They suggested that trapping of the involved segment with distal revascularization may be the treatment of choice. However, some authors reported a high rate of postoperative neurologic morbidity related to lower cranial nerve palsies. $^{22,23}$

Endovascular treatment avoids manipulation of the brain stem, cranial nerves, and cerebellum. Successful endovascular embolization of dissecting PICA aneurysms has been report$\mathrm{ed}^{8,24}$ and is considered relatively safe and reliable. Cellerini et $\mathrm{al}^{11}$ reported successful endovascular treatment of ruptured dissecting PICA aneurysms without parent vessel occlusion in 6 patients. In their series, despite complete embolization of the aneurysms after the procedure, they had 3 early recurrences $(27 \%)$, which represents a relatively high rate of early recurrence given the small series.

The risk of rebleeding is not completely eliminated, even with occlusion of the aneurysm and the parent artery, unless the point of dissection is eliminated. This highlights the difficulty in identifying the exact location of the intimal flap where the dissection begins. As a consequence, the dissection may be incompletely closed. Many authors insist that these lesions are highly unstable. ${ }^{11}$ Choi et $\mathrm{al}^{25}$ reported rerupture following the endovascular treatment of a dissecting aneurysm in the distal anterior inferior cerebellar artery with parent artery preservation. They retreated by parent artery occlusion with detachable coils. We experienced delayed regrowth of a dissecting aneurysm at 4 years and at 5 years 3 months after the initial occlusions of both the aneurysm and the parent artery. This period is significantly longer than other reported cases of rebleeding of dissecting aneurysms. This feature points to the necessity for careful treatment and strict follow-up examination.

The strategy of our treatment is to eliminate flow not only within the aneurysm but also within the dissected PICA segment, thereby decreasing the hemorrhagic risk if the dissection starts proximal to the aneurysm. Nussbaum et $\mathrm{al}^{26} \mathrm{re}-$ ported that PICA occlusion distal to the telovelotonsillar segment generally does not result in brain stem injury and may be reasonably well tolerated with excellent collateral supply to the PICA territory in most patients in their series. Additionally, a distal PICA infarction was not disabling in most of their cases. The risk of brain stem ischemia is limited in cases of permanent occlusion of the first 3 PICA segments, due to the numerous anastomoses of the perforating arteries forming a plexiform network on the medullary surface. ${ }^{27}$ Furthermore, aneurysmal dilation of the dissected arterial segments may be associated with an absence of normal perforating branches, and occlusion of these segments can be performed without further damage to perforating territories. ${ }^{20}$ In one study of endovascular treatment of dissecting aneurysms of the PICA, ${ }^{28}$ they performed balloon test occlusions to evaluate collateral flow from the anterior inferior cerebellar artery, superior cerebellar artery, or accessory PICA. However, a PICA balloon occlusion is not easily performed because of the small caliber and fragility of the vessel.

Technical complications associated with endovascular treatment of peripheral PICA aneurysms seem to be more common compared with intracranial aneurysms in general, which may be due to the tortuous anatomy of the PICA. Some authors reported their experience of rupture during embolization and the results were catastrophic. ${ }^{10,24}$ To prevent pro- 
cedural complications, in cases of small size dissecting aneurysms, we held the tip of the microcatheter proximal to the dissecting aneurysm, formed a couple of loops of the first coil in the parent artery, and then pushed them into the aneurysm, occluding both the dissecting aneurysm and the PICA segment together. This technique may reduce the risk of rupture during the procedure by avoiding manipulation of the catheter and the initial coil loop inside the pseudoaneurysm.

Follow-up angiograms showed 2 cases of recanalization of the occluded parent arteries, 1 case (patient 1) showed recurrence of the parent artery and dissecting aneurysm, and the other case (patient 5) showed recanalization of the parent artery without aneurysm recurrence. For the remaining patients, there were no new clinical symptoms related to the dissection, and follow-up angiograms showed no further change. In the recurrent aneurysm case (patient 1), the patient again complained of severe neck pain, and follow-up MR angiogram showed newly developed intramural hemorrhage around the coil mesh. We embolized the proximal segment of the PICA with the dissecting aneurysm. We suggest that the limited retrograde or antegrade filling of the dissected segment should provide little impetus for enlargement of the aneurysmal dilation or for rebleeding, but we recommend strict follow-up examination with MR angiography and/or digital subtraction angiography. The clinical outcomes in these cases were good to excellent in all patients, even in the 2 patients with initial poor clinical grade on the Hunt and Hess scale.

\section{Conclusions}

Dissecting peripheral PICA aneurysms are rare lesions with a high propensity for bleeding. We describe a series of 6 patients with dissecting peripheral PICA aneurysms presenting with intracranial hemorrhage or intramural hematoma. Five patients were treated with endovascular occlusion of the dissecting aneurysm and the parent artery. The endovascular procedure was well tolerated and avoided the manipulation of the brain stem, cranial nerves, and cerebellum that can be seen with surgery. There was 1 patient with recurrence of the dissected aneurysm, after the initial endovascular occlusion of the aneurysm and parent artery. The patient was endovascularly retreated 2 more times without further recurrence at 2-year follow-up.

For the endovascular treatment of dissecting aneurysms in distal PICA segments, we recommend occlusion of both the dissecting aneurysm and the parent artery to avoid leaving the point of initial intimal tear untreated and to decrease the risk of recanalization and rebleeding. Endovascularly treated patients in our center had excellent clinical outcomes; however, our experience with the case of recanalization emphasizes the need for close follow-up, especially if symptoms recur.

\section{References}

1. Kanou Y, Arita K, Kurisu K, et al. Dissecting aneurysm of the peripheral posterior inferior cerebellar artery. Acta Neurochir (Wien) 2000;142:1151-56

2. Tamano Y, Ujiie H, Hori T. Postoperative hemorrhage due to normal pressure hyperperfusion breakthrough after a trapping of VA-PICA dissecting aneurysm [in Japanese]. No Shinkei Geka 2000;28:257-62

3. Anxionnat R, de Melo Neto JF, Bracard S, et al. Treatment of hemorrhagic intracranial dissections. Neurosurgery 2003;53:289-300; discussion 300-01

4. Yamakawa H, Kaku Y, Yoshimura S, et al. Two cases of dissecting aneurysm of the distal posterior inferior cerebellar artery: possible involvement of segmental mediolytic arteriopathy in the pathogenesis. Clin Neurol Neurosurg 2005;107:117-22

5. Tawk RG, Bendok BR, Qureshi AI, et al. Isolated dissections and dissecting aneurysms of the posterior inferior cerebellar artery: topic and literature review. Neurosurg Rev 2002;26:180-87

6. Kleinpeter $\mathrm{G}$. Why are aneurysms of the posterior inferior cerebellar artery so unique? Clinical experience and review of the literature. Minim Invasive Neurosurg 2004;47:93-101

7. Anxionnat R, Roy D, Bracard S, et al. Dissection of intracranial vertebral arteries revealed by subarachnoidal haemorrhage: report of seven cases. $\mathrm{J} \mathrm{Neu}$ roradiol 1994;21:1-16

8. Halbach VV, Higashida RT, Dowd CF, et al. Endovascular treatment of vertebral artery dissections and pseudoaneurysms. J Neurosurg 1993;79:183-91

9. Tsukahara T, Wada H, Satake K, et al. Proximal balloon occlusion for dissecting vertebral aneurysms accompanied by subarachnoid hemorrhage. Neurosurgery 1995;36:914-19; discussion 919-20

10. Isokangas JM, Siniluoto T, Tikkakoski T, et al. Endovascular treatment of peripheral aneurysms of the posterior inferior cerebellar artery. AJNR Am J Neuroradiol 2008;29:1783-88

11. Cellerini M, Mangiafico S, Ammannati F, et al. Ruptured, dissecting posterior inferior cerebellar artery aneurysms: endovascular treatment without parent vessel occlusion. Neuroradiology 2008;50:315-20

12. Hunt WE, Hess RM. Surgical risk as related to time of intervention in the repair of intracranial aneurysms. J Neurosurg 1968;28:14-20

13. Lister JR, Rhoton ALJ, Matsushima T, et al. Microsurgical anatomy of the posterior inferior cerebellar artery. Neurosurgery 1982;28:170-99

14. Peluso JP, van Rooij WJ, Sluzewski M, et al. Endovascular treatment of symptomatic intradural vertebral dissecting aneurysms. AJNR Am J Neuroradiol 2008;29:102-06

15. Lewis SB, Chang DJ, Peace DA, et al. Distal posterior inferior cerebellar artery aneurysms: clinical features and management. J Neurosurg 2002;97:756-66

16. Mukonoweshuro W, Laitt RD, Hughes DG. Endovascular treatment of PICA aneurysms. Neuroradiology 2003;45:188-92

17. Bradac GB, Bergui M. Endovascular treatment of the posterior inferior cerebellar artery aneurysms. Neuroradiology 2004;46:1006-11

18. Yamaura A. Diagnosis and treatment of vertebra aneurysms. J Neurosurg 1988;69:345-49

19. Ali MJ, Bendok BR, Tawk RG, et al. Trapping and revascularization for a dissecting aneurysm of the proximal posteroinferior cerebellar artery: technical case report and review of the literature. Neurosurgery 2002;51:258-62; discussion 262-63

20. Dinichert A, Rufenacht DA, Tribolet N. Dissecting aneurysms of the posterior inferior cerebellar artery: report of four cases and review of the literature. J Clin Neurosci 2000;7:515-20

21. Fransen P, De Tribolet N. Dissecting aneurysm of the posterior inferior cerebellar artery. Br J Neurosurg 1994;8:381-86

22. Horowitz M, Kopitnik T, Landreneau F, et al. Posteroinferior cerebellar artery aneurysms: surgical results for 38 patients. Neurosurgery 1998;43:1026-32

23. Al-khayat H, Beshay J, Manner D, et al. Vertebral artery-posteroinferior cerebellar artery aneurysms: clinical and lower cranial nerve outcomes in 52 patients. Neurosurgery 2005;56:2-10; discussion 11

24. Yamaura I, Tani E, Yokota M, et al. Endovascular treatment of ruptured dissecting aneurysms aimed at occlusion of the dissected site by using Guglielmi detachable coils. J Neurosurg 1999;90:853-56

25. Choi $\mathrm{CH}$, Cho $\mathrm{WH}$, Choi BK, et al. Rerupture following endovascular treatment for dissecting aneurysm of distal anterior inferior cerebellar artery with parent artery preservation: retreatment by parent artery occlusion with Guglielmi detachable coils. Acta Neurochir (Wien) 2006;148:363-66; discussion 366

26. Nussbaum ES, Madison MT, Myers ME, et al. Dissecting aneurysms of the posterior inferior cerebellar artery: retrospective evaluation of management and extended follow-up review in 6 patients. J Neurosurg 2008;109:23-27

27. Duvernoy HM, ed. Human Brain Stem Vessels. Berlin: Springer; 1999

28. Maimon S, Saraf-Lavi E, Rappaport ZH, et al. Endovascular treatment of isolated dissecting aneurysm of the posterior inferior cerebellar artery. AJNR Am J Neuroradiol 2006;27:527-32 